\title{
A multichannel, portable, "real-time" event encoder-decoder for laboratory and field experiments
}

\author{
REGIS L. MAGYAR and JEFFREY R. FITZSIMMONS \\ University of Florida, Gainesville, Florida 32611
}

\begin{abstract}
An easily constructed, low-cost, portable device is described for observational recording in field studies, or for stimulus programming in the laboratory. The system uses phase-locked loop integrated circuits for recording events in real-time. Multiple events can be simultaneously recorded on a single track of audio cassette tape and subsequently decoded using the same circuit.
\end{abstract}

Observational recording methods available to behavioral researchers range from simple paper-andpencil techniques and mechanical wrist counters to sophisticated electronic systems that code, store, and transfer data directly into computers. These methods are simple and inexpensive, but they (1) provide limited information on the number and kind of behavioral measures that can be made, (2) are inadequate for relatively complex behavioral analysis, and (3) often require frequent shifts in the researcher's attention from observing behaviors to recording them. On the other hand, the development of more sophisticated electronic systems (see Sidowski, 1977) has provided remedies to these problems by allowing an observer to continuously record the occurrences and durations of several behaviors, thus providing for subsequent sequential and time-series analysis of the data. Unfortunately, the advantages provided by the more sophisticated systems are presently offset by the relatively high cost of implementing the systems, the difficulty and complexity of construction of the electronic circuitry, and the need for peripheral equipment (e.g., computer interfacing and the hardware and software required for decoding and analyzing data) not commonly or inexpensively available to a majority of researchers in the field. The level of analysis in behavioral research is, in part, determined by the descriptive adequacy provided by the measurement technique. The development of simple, yet inexpensive, recording systems that incorporate many of the desirable features found in more complex systems would be of practical value to researchers with limited budgets and equipment.

In general, attempts to bridge this gap have largely been unsuccessful. Several investigators have devised keyboard event-recording systems that record multiple

Requests for reprints should be sent to Regis L. Magyar, Department of Psychology, University of Florida, Gainesville, Florida 32611 . entries on strip charts (Baker \& Whitehead, 1972) or simply encode them as tones on audio tape (Dawkins, 1971; Whittaker, 1973) without providing an automated means for decoding them. Devices that included decoding systems have been limited to single-channel data capabilities (Kintz, 1965; Masterson, 1965; Wolach, Roccaforte, \& Breuning, 1975), involved complex digital coding circuitry necessitating a separate decoder unit (Hopkins, 1972; Kellenyi \& Buzsaki, 1977), or required relatively complex interfacing systems (Sackett, Stephenson, \& Ruppenthal, 1973). Despite sometimes costly and elaborate circuitry, a number of these devices are unable to encode and subsequently decode the simultaneous occurrence of events recorded or stored on magnetic tape. The development of more sophisticated recording systems (e.g., Fitzpatrick, 1977; Stephenson \& Roberts, 1977) employing advanced electronic circuitry and microprocessor technology have easily overcome these difficulties. However, these systems are relatively (but not exorbitantly) expensive at present, and they require computer transcription of data.

This paper describes a relatively simple device with wide applicahility to both laboratory and field experiments. The unit may be used in conjunction with any portable cassette recorder as a multichannel event encoder-decoder for observational studies, and it incorporates many desirable qualities found in more complex and expensive devices. It is hand held, battery operated, simple to use, inexpensive, and easily constructed. Because of its unique design, multiple events can be simultaneously encoded and subsequently decoded in real-time using a single track of audio tape. The unit is compatible with solid state and electromechanical programming equipment, which allows the circuit to be used as a stimulus programmer.

\section{GENERAL DESCRIPTION}

The Signetics 567 phase-locked loop (PLL) in tegrated circuit (IC) is the basis of the present design. The PLL 
compares the frequency of a signal appearing at its input to an internally generated signal (center frequency $=\mathrm{f}_{0}$ ). When the frequencies of the two signals correspond, an internal comparator in the PLL causes the output voltage level to change states (see Cohen, 1978; Hoats, 1975).

The way in which the PLL may be used as an event recording system or as a stimulus programmer is exemplified in Figure 1, which shows the circuit diagram for a simple two-channel event encoder-decoder. Component values for the circuit are shown in Table 1. Each IC is used as a separate channel that converts the occurrence of a discrete event into a distinct audio frequency that is recorded on audio tape. The same IC that encodes the event subsequently decodes it. In the recording mode, pressing one or both of the pushbuttons (PB-1, PB-2) allows the internal oscillator output from each IC to be gated out to a common output jack $\left(\mathrm{J}_{2}\right)$. The oscillation frequency $\left(f_{0}\right)$ of each IC is determined by the R-C timing circuit across Pins 5 and 6 (for IC-1, R-2 and C-3; for IC-2, R-4 and C-6). The formula for computing each channel's frequency is given in Table 2 . In general, these programmed frequencies should be separated by at least $300 \mathrm{~Hz}$ to insure reliable separation of each channel.

Depending on the buttons pressed during coding operations, one or more frequencies appear across the output jack $\left(\mathrm{J}_{2}\right)$, and are then fed into the microphone input jack of any cassette or reel-to-reel tape recorder. Input voltage to the recorder is limited by resistor R-7; diodes D-1 and D-2 insure against signal back paths to each PLL.

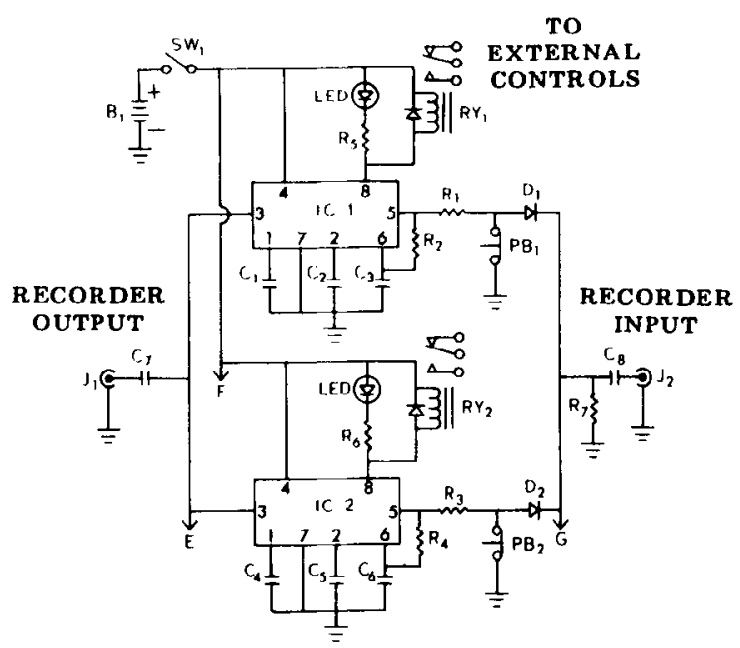

Figure 1. Circuit diagram of a two-channel event encoderdecoder. Component values are shown in Table 1. Events from each channel are converted into tones (see Table 2) and recorded on audio tape via J-2. Recorded data are converted into discrete relay closures and/or LED indicator flashes when the taperecorder output is played back through J-1. Connection points for added event channels are made at Points F, F, and G (see text).
Table 1

Component Values for the Two-Channel Encoder-Decoder Circuit Shown in Figure 1

Resistors

$\mathrm{R}-1, \mathrm{R}-3=100 \mathrm{kohm}$
$\mathrm{R}-2=5.6 \mathrm{~K}$
$\mathrm{R}-4=6.8 \mathrm{~K}$
$\mathrm{R}-5, \mathrm{R}-6=2.2 \mathrm{~K}$.
$\mathrm{C}-1, \mathrm{C}-4=4.7$
$\mathrm{C}-2, \mathrm{C}-5=2.2$
$\mathrm{C}-3, \mathrm{C}-6=.1$
$\mathrm{C}-7, \mathrm{C}-8=.47$
$\quad$ Semiconductors
D-1, D-2 = 1 N914
IC-1, IC-2 = Signetics-567 phase-lock loop
integrated circuit (or equivalent)
$\quad$ Miscellaneous
PB-1, PB-2 = momentary contact SPST
pushbutton (normally closed)
SW-1 = SPST miniature toggle
LEDs = light-emitting diodes (20 to $40 \mathrm{~mA}$ )
B-1 = 9-V transistor radio-type battery
Connectors J-1, J-2 = miniature phone jacks
RY-1, RY-2 = IC-compatible reed relays or
any low-current 9-V relay

Note-All resistors are $.25 \mathrm{~W}$; all capacitors are in microfarads, $10 . \mathrm{V} d \mathrm{c}$ rating.

The multichannel data recorded on a single track of audio tape are easily decoded by playing back the tape recording via an earphone jack through the input jack $\left(J_{1}\right)$ of the encoder-decoder. Each PLL monitors the tape-recorder output at $\mathrm{Pin} 3$. When the appropriate tone frequency on the tape recording matches the internally generated frequency of a given PLL, the output voltage level of that IC (Pin 8 ) changes from its normally high state $(+9 \mathrm{~V})$ to a low state $(0 \mathrm{~V})$. This changed output level is maintained as long as the appropriate matched frequency is detected at Pin 3. Since the 567-IC can handle up to $100 \mathrm{~mA}$ of current, it is capable of driving either solid state logic devices or low-powered loads. In the present design, each channel output (Pin 8) directly drives a light-emitting diode (LED) indicator lamp, as well as a low-voltage relay for each tone decoded. Since relays are now available in IC form (dual-in-line packages with builtin diodes for back-voltage protection), they can be easily added to the working circuitry and require very little space in the constructed unit.

Additional channels are easily added to the circuit shown in Figure 1 at Points E, F, and G. The positive terminal of the battery (F) is connected to Pin 4 of each IC, while the input of each added channel (Pin 3 ) is connected at Point $E$. The circuit component values for each added PLL are identical to those specified for each of the two channels shown in Figure 1, except for the value of the resistor across Pins 5 and 6, which determines the internal oscillation frequency of each IC. 
Table 2

Computation of Center Frequency $\left(\mathrm{F}_{\mathrm{o}}\right)$ for Each Tone Encoder-Decoder Channel Shown in Figure 1

$\frac{\text { Channel 1 (IC-1) }}{\mathrm{F}_{1}=\frac{1}{(\mathrm{R}-2)(\mathrm{C}-3)}=\frac{1}{(5.6 \mathrm{~K})(.1 \mathrm{microF})} \cong 1,800 \mathrm{~Hz} \quad \mathrm{~F}_{2}=\frac{1}{(\mathrm{R}-4)(\mathrm{C}-6)}=\frac{1}{(6.8 \mathrm{~K})(.1 \mathrm{microF})} \cong 1,500 \mathrm{~Hz}}$

Note-Resistor values $(R \cdot 2, R-4)$ are in ohms; capacitor values $(C \cdot 3, C \cdot 6)$ are in microfarads.

For example, a third channel with a desired center frequency of $1,000 \mathrm{~Hz}$ requires a $10-\mathrm{kohm}$ resistor across Pins 5 and 6 of the added IC (see formulas in Table 2). The oscillator output of the added PLL is tapped at Pin 5 through the 100-kohm resistor, normally closed pushbutton, and diode complex, and is connected to the common output of the circuit at Point G. The number of channels that can be added depends largely on the quality of the tape recorder and the audio tape used. While most cassette recorders can accommodate between five and seven channels, it is important to consider the frequency-response characteristics of the recorder, as well as the level of wow and flutter in the tape drive mechanism.

A complete three-channel encoder-decoder circuit, including battery, jacks, and pushbuttons can be conveniently mounted within a small chassis $(3 \times 4 \times 1.5$ in. $)$, allowing hand-held operation. A single coaxial cable with a miniature male phone jack attached to each end links the encoder-decoder circuit to the cassette recorder. Only one mode of operation (i.e., record or decode) should be used at a time. Since most batteryoperated recorders provide a remote start-stop jack, an optional latching-type pushbutton switch may be added to the encoder-decoder unit to start or stop the tape transport motor of the recorder during operation.

The circuit operates on one $9 . \mathrm{V}$ battery and consumes very little current, $10 \mathrm{~mA}$ plus relay current requirements. Since the unit is totally independent of specific peripheral equipment, the relay contacts can be used to drive any medium-power external device during decoding. These may include electromechanical counters, running-time meters, and an event or cumulative recorder. Solid state logic can be driven directly from the voltage output of Pin 8 for each PLL, thus eliminating the need for relays.

\section{$\operatorname{COST}$}

Parts for the circuit can be obtained from any electronic supply dealer or through mail-order wholesale dealers at a considerable savings. The cost of each 567-PLL ranges from $\$ 1$ to $\$ 2$. A three-channel encoderdecoder can be constructed for under $\$ 20$; portable cassette recorders range from $\$ 20$ to $\$ 30$.

\section{APPLICATIONS}

A primary advantage of this recording system is its portable and remote data collecting capabilities. It provides an affordable, yet simple, means for collecting continuous measurements of multiple events in field observational studies. Because of the system's unique decoding abilities, data recorded in field settings can be automatically analyzed in the laboratory by using conventional and commonly available electromechanical programming circuitry. For example, durations of given observed behaviors or events can be obtained by depressing a pushbutton on the encoder for the length of time an event was observed. During data analysis, the output relay contacts of the decoder circuit can be used to operate running-time meters to accumulate the durations. Similarly, the occurrences, as well as the local or overall rates of observed behaviors, can be analyzed by interfacing the decoder outputs into programmed electromechanical steppers, timers, and counters. Since data are recorded, stored, and decoded in real-time, an investigator can also analyze the relationships between the occurrences of stimulus events and observed behaviors (latencies, reaction times, etc.). The temporal sequence of several events can be studied by simply displaying these data as events on a cumulative recorder or a multichannel recorder. In general, the present recording system allows for alternative ways to achieve adequate measurements in the absence of sophisticated computer systems.

The encoder-decoder circuit is also useful in a number of operant conditioning experiments. By substituting the normally closed pushbutton switches with normally closed relay contacts from pulse formers in the experimental programming circuitry, a subject's responses (leverpresses, keypecks, etc.) can be recorded by one channel, and corresponding scheduled events (i.e., discriminative stimuli and reinforcements) can be recorded by other channels. The data collected during an experimental session can be stored on a single track of audio tape for subsequent reanalysis or playback into a cumulative recorder at a later time (c.f. Wolach et al., 1975). In addition, data recorded during experimental sessions may be used to conduct yoked control studies. For example, a tape recording of the exact reinforcement frequency produced by a subject responding under a schedule of reinforcement can be used to subsequently program reinforcer deliveries independent of the subject's behavior in later sessions. This procedure allows for a comparison of response rates during response-dependent and response-independent schedules equated for reinforcement frequency.

Finally, the system can also be used in studies of human learning and memory, which often require the 
presentation of both auditory and visual stimuli. Multiple events can be encoded on one channel of a stereo tape deck and synchronized with auditory stimuli recorded on the other channel. During playback, the system decodes the events and operates several slide projectors that provide the necessary visual stimuli.

\section{REFERENCES}

Baker, J. G., \& Whitehead, G. A portable recording apparatus for rating behavior in free-operant situations. Journal of Applied Behavior Analysis, 1972, 5, 191-192.

CoHEN, H. How phase-locked loops work. Popular Electronics: 1978 Electronic Experimenter's Handbook. New York: ZiffDavis, 1978.

DAwkins, R. A cheap method of recording behavioral events for direct computer access. Behavior, 1971, XL, 162-173.

Fitzpatrick, L. Automated data collection for observed events. Behuvior Research Methods \& Instrumentation, 1977, 9, 447.451.

HoATS, D. L. A pair of electronic devices for detecting restrictedband audio and ultrasonic frequencies. Behavior Research Methods \& Instrumentation, 1975, 7, 542-544.

Hopkins, R. M. A digital programmer for use with audio tape decks. Behavior Research Methods \& Instrumentation, 1972, 4, 317-319.
Kellenyt, L., \& Buzsaki, G. Simple device for the tape recording of complex behavioral situation on one track. Physiology \& Behavior, 1977, 19, 449-450.

Kintz, R. T. The tape recorder as a programmer. Joumal of the Experimental Analysis of Behavior, 1965, 8, 294.

MAsterson, F. A. Yoked control without a duplicate apparatus: A response record-playback system. Joumal of the Experimental Analysis of Behavior, 1965, 8, 395-396.

Sackett, G. P., Stephenson, E., \& Ruppenthal, G. C. Digital data acquisition systems for observing behavior in laboratory and field setting. Behavior Research Methods \& Instrumentation, $1973,5,344-348$.

Sidowski, J. B. Observational research: Some instrumented systems for scoring and storing behavioral data. Behavior Research Methods \& Instrumentation, 1977, 9, 403-404.

Stephenson, G. R., \& Roberts, T. W. The SSR system 7: A general encoding system with computerized transcription. Behavior Research Methods \& Instrumentation, 1977. 9. 434-441.

WHITTAKER, R. A note on a multi-movement, on-line behavior recorder. Joumal of Applied Behavior Analysis, 1973, 6, 721-723.

Wolach, A. H., Roccaforte, P., \& Breuning, S. E. Tape recording data for a cumulative recordet and for programming interval schedules. Behavior Research Methods \& Instrumentation, 1975, 7, 545-546.

(Received for publication May 16, 1978; revision accepted August 24, 1978.) 